\title{
Cell-wall thickness: possible mechanism of acriflavine resistance in meticillin-resistant Staphylococcus aureus
}

\begin{abstract}
Correspondence
Jun-ichi Yamagishi

junichi-yamagishi@ds-

pharma.co.jp
\end{abstract}

Received 13 June 2008

Accepted 27 October 2008

\author{
Mako Kawai, ${ }^{1}$ Sakuo Yamada, ${ }^{2,3}$ Ai Ishidoshiro, ${ }^{4}$ Yoshihiro Oyamada, ${ }^{5}$ \\ Hideaki Ito $^{6}$ and Jun-ichi Yamagishi ${ }^{1}$ \\ ${ }^{1}$ Microbiological Control Laboratories, Technology Research and Development Center, Dainippon \\ Sumitomo Pharma Co. Ltd, 5-51 Ebie 1-chome Fukushima-ku, Osaka 553-0001, Japan \\ ${ }^{2}$ Department of Microbiology, Kawasaki Medical School, 577 Matsushima, Kurashiki, Okayama \\ 701-0192, Japan \\ ${ }^{3}$ Department of Clinical Nutrition, Kawasaki University of Medical Welfare, 288 Matsushima, \\ Kurashiki, Okayama 701-0193, Japan \\ ${ }^{4}$ Quality Assurance Group, Ibaraki Manufacturing Plant, Dainippon Sumitomo Pharma Co. Ltd, 3-45 \\ 1-chome Kurakakiuchi, Ibaraki, Osaka 567-0878, Japan \\ ${ }^{5}$ Pharmacology Research Laboratories, Dainippon Sumitomo Pharma Co. Ltd, Enoki, 33-94 Suita, \\ Osaka 564-0053, Japan \\ ${ }^{6}$ Pharmacovigilance, Dainippon Sumitomo Pharma Co. Ltd, 6-8 Doshomachi 2-Chome, Chuo-ku, \\ Osaka 541-0045, Japan
}

\begin{abstract}
Acriflavine resistance in the clinical meticillin-resistant Staphylococcus aureus isolate KT24 was found not to be mediated by multidrug efflux pumps encoded by qacA/B, smr, qacE, qacG, qacH, qacJ or norA. Early uptake and accumulation of ethidium bromide in MRSA KT24 was significantly lower than that in a susceptible strain, although the efflux rates were similar. Therefore, a permeability barrier in MRSA KT24 may be the conceivable mechanism of acriflavine resistance. Interestingly, it was found that MRSA KT24 had a significantly thickened cell wall, and that cellwall thickness increased gradually during bacterial growth. In contrast, cell size and surface area in MRSA KT24 were not different from those in the susceptible strain. Moreover, MRSA KT24 exposure to sub-MIC concentrations of acriflavine resulted in a thicker cell wall. These results indicate that cell-wall thickness may be responsible for acriflavine resistance in $S$. aureus.
\end{abstract}

\section{INTRODUCTION}

Meticillin-resistant Staphylococcus aureus (MRSA) is a clinically significant bacterial pathogen because it is resistant to a variety of antibiotics and causes nosocomial infections worldwide. The ability of Staphylococcus spp. to adhere and form multilayered biofilms on host tissues and other surfaces is one of the important mechanisms by which these bacteria are able to persist in some diseases (Beenken et al., 2004), making the prevention of nosocomial disease dissemination difficult.

It is generally believed that bacteria rarely acquire resistance to antiseptic agents as these agents act at several target sites (McDonnell \& Russell, 1999; Russell, 2003). However, MRSA has been reported to be resistant to various antiseptics, including benzalkonium chloride and chlorhexidine digluconate (Noguchi et al., 1999; Suller \&

Abbreviation: MRSA, meticillin-resistant Staphylococcus aureus.
Russell, 1999; Mayer et al., 2001). In addition, a growing number of species have demonstrated cross-resistance not only to other antiseptic agents but also to antibiotics.

Although inactivation and target alteration are known mechanisms for resistance to antiseptic agents, they are comparatively rare. Resistance typically results from cellular changes that negatively affect the accumulation of antiseptic agents, including cell envelope changes that limit uptake or expression of efflux mechanisms (Poole, 2002; Gilbert \& McBain, 2003; Russell, 2003; Piddock, 2006). In $S$. aureus, qacA, qacB, smr, nor $A$ and $m d e A$, which encode multidrug-transporter proteins, have been identified as genes that confer resistance to antiseptic agents (Rouch et al., 1990; Alam et al., 2003a, b; Huang et al., 2004). The nor $A$ and $m d e A$ genes are located on the chromosome of $S$. aureus, whilst the qacA, qacB and $s m r$ genes have been found mainly on plasmids (Piddock, 2006). The proteins encoded by $q a c A / B$ and $s m r$ belong to a major facilitator 
superfamily and small multidrug-resistance family, respectively. Additional resistance genes, such as qacG, $q a c H$, qacE and qacj, have also been identified (Bjorland et al., 2003).

Acriflavine has been used for a variety of biological and biochemical purposes, especially as an antiseptic agent possessing a quaternary ammonium structure. It is well known that one mechanism of acriflavine resistance is due to overexpression of efflux pump genes. However, other mechanisms are poorly understood. In this study, we examined the distribution of well-known multidrug efflux pump genes in clinical isolates and investigated the mechanism of acriflavine resistance in the MRSA isolate KT24, which does not possess efflux pump genes.

\section{METHODS}

Reagents. Benzalkonium chloride was purchased from ICN Biomedicals. Ampicillin was from Calbiochem-Novabiochem, and cefalotin, vancomycin and chloramphenicol were from SigmaAldrich. Sparfloxacin was synthesized at Dainippon Sumitomo Pharma. Other reagents were purchased from Wako-Pure Chemical Industries unless otherwise indicated.

Bacterial strains and culture conditions. A total of 38 MRSA isolates were collected at Kitazato University Hospital, Japan, between 1999 and 2000. S. aureus 209P, S. aureus ATCC 12600, S. aureus ATCC 29210, S. aureus RN4220 and S. aureus RN2677 were used as susceptible reference strains. The $S$. aureus strains used were cultured aerobically in soybean casein digest broth (SCDB; Nihon Pharmaceutical).

Determination of antimicrobial susceptibility. MICs were determined by a twofold serial agar dilution method using Mueller-Hinton agar (BD) as recommended by CLSI (2003) (formerly the National Committee for Clinical Laboratory Standards). To assess NorA efflux pump activity, MICs were determined in the presence of reserpine (final concentration $20 \mu \mathrm{g} \mathrm{ml}^{-1}$ ).

Preparation of genomic DNA. S. aureus genomic DNA was prepared as described by Hudson \& Curtiss (1990), except that $100 \mu \mathrm{g}$ mutanolysin $\mathrm{ml}^{-1}$ was replaced by $50 \mu \mathrm{g}$ lysostaphin $\mathrm{ml}^{-1}$.
PCR amplification. Amplicons corresponding to the antisepticresistance genes $q a c A / B, s m r, q a c E$, qacG, qacH and qacJ were amplified by PCR with the primer sets shown in Table 1. PCR was performed using Ex Taq polymerase (TaKaRa). Reactions were carried out for 30 cycles of $15 \mathrm{~s}$ at $94{ }^{\circ} \mathrm{C}, 30 \mathrm{~s}$ at $60{ }^{\circ} \mathrm{C}$ and $1 \mathrm{~min}$ at $72{ }^{\circ} \mathrm{C}$. The PCR products were analysed by agarose gel electrophoresis.

Accumulation and efflux of ethidium bromide. Ethidium bromide uptake and efflux were determined using a modified fluorescence technique (Kaatz et al., 2000). Organisms were grown overnight in SCDB, and a $2 \%(\mathrm{v} / \mathrm{v})$ inoculum of this culture was added to $100 \mathrm{ml}$ Mueller-Hinton broth (MHB; BD). The cells were harvested at an $\mathrm{OD}_{660}$ of 0.7 , washed in ice-cold $\mathrm{MHB}$ and resuspended in $\mathrm{MHB}$ to a final $\mathrm{OD}_{660}$ of 0.7 . For the ethidium bromide uptake assay, the cell suspension was maintained at $37^{\circ} \mathrm{C}$ for 2-3 min and ethidium bromide (final concentration $10 \mu \mathrm{g} \mathrm{ml}^{-1}$ ) was added. The fluorescence of aliquots of the mixture was determined at frequent intervals (excitation wavelength $545 \mathrm{~nm}$; emission wavelength $590 \mathrm{~nm}$ ) using a luminescence spectrometer (Thermo Fisher Scientific). For the ethidium bromide efflux assay, ethidium bromide (final concentration $10 \mu \mathrm{g} \mathrm{ml}^{-1}$ ) and carbonyl cyanide $m$-chlorophenylhydrazone (final concentration $100 \mu \mathrm{M}$ ) were added to the cell suspension. After $30 \mathrm{~min}$ incubation at $37^{\circ} \mathrm{C}$, the cells were pelleted and resuspended in fresh MHB. The fluorescence of aliquots of the mixture was determined at frequent intervals as above.

Electron microscope analysis. After incubation at $37^{\circ} \mathrm{C}$, bacterial cells were harvested and washed twice with Dulbecco's PBS.

For transmission electron microscopy, the cells were collected into a pellet and fixed with $2.5 \%$ glutaraldehyde, followed by $1 \% \mathrm{OsO}_{4}$. The samples were dehydrated by passing through an ethanol series and then embedded in Spurr's Epon. Ultrathin sections were obtained using an ultramicrotome with a diamond knife and examined under a JEOL JEM-2000EXII electron microscope at $80 \mathrm{kV}$.

For scanning electron microscopy, a drop of the bacterial cell suspension was mounted on a coverslip and fixed with $2.5 \%$ glutaraldehyde, followed by $1 \% \mathrm{OsO}_{4}$. The samples were stained with $1 \%$ tannic acid and fixed with $1 \% \mathrm{OsO}_{4}$. After dehydration using an ethanol series and $t$-butyl alcohol, the samples were dried and coated using platinum, and then examined using a JEOL JSM-6340F field emission scanning electron microscope at $15 \mathrm{kV}$. Cell-wall thickness was determined using photographs taken at a final magnification of $\times 10000$. More than ten cells that had been cut through the cell equator were chosen for each strain, and cell-wall thickness was determined at four sites for each cell.

Table 1. Primers used for PCR amplification

\begin{tabular}{|c|c|c|}
\hline Target gene & Sequence $\left(5^{\prime} \rightarrow 3^{\prime}\right)$ & Reference \\
\hline \multirow[t]{2}{*}{$q a c A / B$} & GCAGAAAGTGCAGAGTTCG & Rouch et al. (1990) \\
\hline & CCAGTCCAATCATGCCTG & \\
\hline \multirow[t]{2}{*}{ smr } & GCCATAAGTACTGAAGTTATTGGA & Sasatsu et al. (1989) \\
\hline & GACTACGGTTGTTAAGACTAAACCT & \\
\hline \multirow[t]{2}{*}{$q a c E$} & GCAATAGTTGGCGAAGTAATCG & This study \\
\hline & GGCTGTAATTATGACGACGCC & \\
\hline \multirow[t]{2}{*}{$q a c G$} & TCAATTGCAACAGAAATAATCGGA & This study \\
\hline & GATTATTGTTGTTAATACTAATCC & \\
\hline \multirow[t]{2}{*}{$q a c H$} & CAATAGTCAGTGAAGTAATAGG & This study \\
\hline & AACAATTGTTGTTAATACTAATCCT & \\
\hline \multirow[t]{2}{*}{$q a c J$} & GCGATTATAACTGAAATAATAGGA & This study \\
\hline & AACTATTGTTGTAAGAACTAGTCC & \\
\hline
\end{tabular}




\section{RESULTS AND DISCUSSION}

In this study, we examined susceptibility to acriflavine and the distribution of antiseptic-resistance genes in clinically isolated MRSA. MIC values of antiseptic agents for the susceptible strains S. aureus ATCC 12600, S. aureus ATCC 29210, S. aureus RN4220, S. aureus RN2677 and S. aureus 209P were equal within an error range. Thus we decided to use S. aureus $209 \mathrm{P}$ as a representative susceptible strain. The acriflavine MIC value for $S$. aureus $209 \mathrm{P}$ was $1 \mu \mathrm{g}$ $\mathrm{ml}^{-1}$, whilst those for all of the MRSA clinical isolates were four or more times higher, ranging from 4 to $>128 \mu \mathrm{g}$ $\mathrm{ml}^{-1}$. Based on the acriflavine MIC value for $S$. aureus 209P, MRSA isolates were classified into three groups: a 'high-level resistant group' (MIC $>64 \mu \mathrm{g} \mathrm{ml} \mathrm{ml}^{-1}$ ), an 'intermediate-level resistant group' (MIC $16-32 \mu \mathrm{g} \mathrm{ml}^{-1}$ ) and a 'low-level resistant group' (MIC 4-8 $\mu \mathrm{g} \mathrm{ml}^{-1}$ ). The frequency as detected by PCR of the $q a c A / B$ and $s m r$ genes, which are the most common plasmid-mediated resistance genes, is summarized in Table 2. S. aureus 209P was not found to possess the $q a c A / B$ or $s m r$ gene by PCR. However, overall, $q a c A / B$ and $s m r$ were detected in $55 \%(21 / 38)$ and $21 \%(8 / 38)$, respectively, of all of the MRSA isolates examined. MRSA isolates carrying both the qacA/B and $s m r$ genes represented $13 \%(5 / 38)$ and neither $q a c A / B$ nor $s m r$ was detected in $11 \%(4 / 38)$ of all isolates. Thus more than $80 \%$ of the MRSA isolates possessed plasmid-borne resistance genes. The $q a c A / B$ gene in the high-level and intermediate-level resistant groups was detected at a significantly higher rate than in the low-level resistant group. In contrast, the acriflavine MIC value for MRSA isolates carrying both the $q a c A / B$ and $s m r$ genes was not higher than that for isolates carrying the $q a c A / B$ or $s m r$ gene. These findings, which are in agreement with those of a previous study (Mayer et al., 2001), suggest that the qacA/ $B$ gene is widely distributed in MRSA.

As previous studies have indicated that reserpine is capable of inhibiting the NorA pump (Aeschlimann et al., 1999; Gibbons et al., 2003), NorA expression might account for the fourfold reduction in acriflavine MIC in the presence of reserpine. After the addition of reserpine, acriflavine MICs decreased by $42 \%(16 / 38)$ overall in the MRSA isolates.
Therefore, the existence of a plural multidrug efflux system did not increase susceptibility to acriflavine in MRSA.

Among the 38 MRSA clinical isolates, MRSA isolate KT24 was found to exhibit high-level resistance to acriflavine (MIC $128 \mu \mathrm{g} \mathrm{ml}^{-1}$ ) without possessing the $q a c A / B$ and $s m r$ genes. Additional resistance-related genes, such as qacE, qacG, qacH and qacJ (Paulsen et al., 1996; Alam et al., 2003a, b), were also not detected by PCR in MRSA KT24 (data not shown). Moreover, susceptibility determination with reserpine revealed no NorA expression in MRSA KT24. Therefore, the high-level resistance of MRSA KT24 to acriflavine may be caused by an unknown mechanism.

The MICs of a series of antiseptic agents for MRSA KT24 and the susceptible strain S. aureus 209P are shown in Table 3. The MIC values of some antiseptic agents, such as acriflavine, acrinol and ethidium bromide, for MRSA KT24 were significantly higher than those for the susceptible strain S. aureus 209P. Acriflavine, benzalkonium chloride, benzethonium chloride, hexadecyltrimethylammonium bromide and ethidium bromide all possess a quaternary ammonium structure. However, MRSA KT24 showed high-level resistance to acriflavine (MIC $128 \mu \mathrm{g} \mathrm{ml}^{-1}$ ) but low-level resistance to benzalkonium chloride (MIC $4 \mu \mathrm{g} \mathrm{ml}{ }^{-1}$ ). For other compounds with different structures, MRSA KT24 susceptibility varied greatly, with, for example, MIC values of $>128 \mu \mathrm{g} \mathrm{ml}^{-1}$ for acrinol and $2 \mu \mathrm{g}$ $\mathrm{ml}^{-1}$ for chlorhexidine gluconate. However, MRSA KT24 was susceptible to triclosan, which contains bisphenols.

In addition to the antiseptic agents mentioned above, a number of antimicrobial agents with different targets and mechanisms of action, such as inhibitors of cell-wall synthesis, protein synthesis and DNA synthesis, were also evaluated. Although MRSA KT24 was resistant to some antimicrobial agents, such as erythromycin, norfloxacin and tetracycline, it was susceptible to vancomycin, chloramphenicol, rifampicin and novobiocin. In general, MRSA KT24 showed resistance to various kinds of antiseptic and antimicrobial agents with different structures and mechanisms of action. For the purpose of this study, we examined further the mechanism underlying MRSA KT24 resistance to acriflavine.

Table 2. Distribution of the antiseptic drug efflux genes qacA/B and $s m r$, and NorA expression, in 38 isolates of MRSA

Results are shown as numbers of isolates, with the percentage detection rate for each group shown in parentheses.

\begin{tabular}{|c|c|c|c|c|c|c|c|}
\hline \multirow{2}{*}{$\begin{array}{l}\text { Acriflavine } \\
\text { susceptibility group }\end{array}$} & \multirow{2}{*}{$\begin{array}{l}\text { MIC of acriflavine } \\
\quad\left(\mu \mathrm{g} \mathrm{ml}^{-1}\right)\end{array}$} & \multirow{2}{*}{$\begin{array}{c}\text { No. of } \\
\text { isolates }\end{array}$} & \multicolumn{4}{|c|}{ Gene(s) } & \multirow[t]{2}{*}{ NorA expression } \\
\hline & & & $q a c A / B$ & $s m r$ & $q a c A / B+s m r$ & $\mathrm{ND}^{\dagger}$ & \\
\hline I & $>64$ & 15 & $12(80)$ & $0(0)$ & $0(0)$ & $3(20)$ & $3(20)$ \\
\hline II & $16-32$ & 16 & $8(50)$ & $5(31)$ & $3(19)$ & $0(0)$ & $10(63)$ \\
\hline III & $4-8$ & 7 & $1(14)$ & $3(43)$ & $2(29)$ & $1(14)$ & $3(43)$ \\
\hline
\end{tabular}

${ }^{*}$ Group I, high-level resistance group; group II, intermediate-level resistance group; group III, low-level resistance group.

$\dagger \mathrm{ND}$, Neither $q a c A / B$ nor $s m r$ was detected. 
Table 3. Antimicrobial susceptibility of MRSA KT24 and the susceptible strain S. aureus 209P

\begin{tabular}{|lcc|}
\hline \multirow{2}{*}{ Agent } & \multicolumn{2}{c|}{ MIC $\left(\boldsymbol{\mu g} \mathbf{~ m l}^{-\mathbf{1}}\right)$} \\
\cline { 2 - 3 } & KT24 & $\mathbf{2 0 9 P}$ \\
\hline Acriflavine & 128 & 1 \\
Acrinol & 128 & 8 \\
Benzalkonium chloride & 4 & 1 \\
Benzethonium chloride & 16 & 1 \\
Chlorhexidine gluconate & 2 & 0.5 \\
Ethidium bromide & 64 & 0.5 \\
Hexadecyltrimethylammonium bromide & 8 & 0.5 \\
Rhodamine 6G & 2 & 0.25 \\
Triclosan & 0.063 & 0.063 \\
Ampicillin & 0.25 & 0.063 \\
Cefalotin & 0.5 & 0.125 \\
Chloramphenicol & 4 & 2 \\
Erythromycin & $>128$ & 0.125 \\
Gentamicin & 16 & 0.063 \\
Norfloxacin & 64 & 0.125 \\
Novobiocin & $<0.063$ & $<0.063$ \\
Rifampicin & $<0.063$ & $<0.063$ \\
Sparfloxacin & 8 & 0.063 \\
Tetracycline & 32 & 0.125 \\
Vancomycin & 1 & 0.5 \\
& & \\
\hline
\end{tabular}

To assess bacterial efflux activity and cell permeability, we examined ethidium bromide accumulation in MRSA KT24 and S. aureus 209P. In S. aureus 209P cells, ethidium bromide uptake was rapid, with effective accumulation; in contrast, early uptake and accumulation of ethidium bromide in MRSA KT24 cells was significantly lower (Fig. 1a). However, the efflux rate of ethidium bromide in MRSA KT24 cells was similar to that in S. aureus 209P (Fig. 1b). It has been reported that active efflux pumps play a major role in micro-organism resistance to antiseptic agents (Gilbert \& McBain, 2003). However, our results showed that uptake and accumulation of ethidium bromide in MRSA KT24 cells, rather than efflux activity, resulted in reduced susceptibility to the antiseptic agent. It is therefore possible that a permeability barrier is one of the mechanisms underlying MRSA KT24 resistance to acriflavine.

We examined the hydrophobicity of MRSA KT24 following the assay described previously by Rosenberg \& Rosenberg (1981) and compared it with that of S. aureus 209P. Although about $80 \%$ of $S$. aureus 209P cells remained in the aqueous phase after mixing with $n$-octane, only approximately $30 \%$ of MRSA KT24 cells remained in the aqueous phase (data not shown). Based on the criteria for distinguishing between hydrophilic and hydrophobic properties described by Yamada \& Matsumoto (1990), MRSA KT24 was found to have a hydrophobic surface, whilst S. aureus 209P cells were hydrophilic. Dominant hydrophobicity is probably due to proteins and proteinassociated molecules localizing at the surface of the
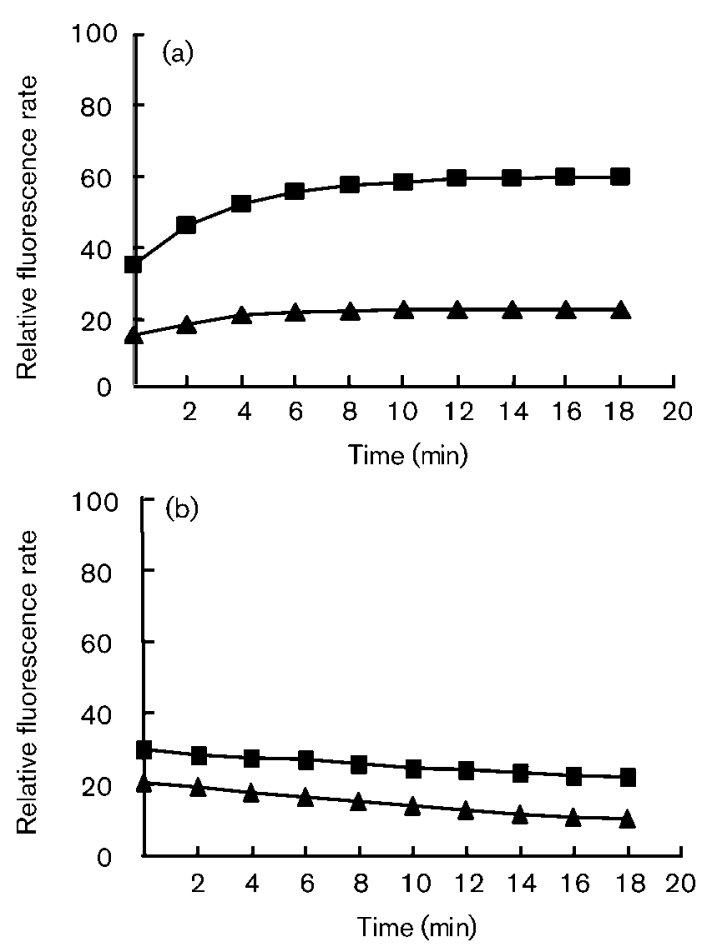

Fig. 1. Uptake and accumulation (a) and efflux (b) of ethidium bromide. $\mathbf{\square}$. aureus 209P; $\mathbf{\Delta}$, MRSA KT24.

organism and is believed to arise from a lack of teichoic acid, protein A or coagulase production (Reifsteck et al., 1987). Therefore, the surface proteins of MRSA KT24 may be different from those of S. aureus 209P. Wadström (1990) reported that cell-surface hydrophobicity may affect bacterial susceptibility to antimicrobial and antiseptic agents.

To clarify additional potential resistance mechanisms, the morphological characteristics of the cells were observed by electron microscopy. Stationary-phase cells of MRSA KT24 were compared with those of S. aureus 209P based on scanning electron microscopy analysis (Fig. 2a). No differences in individual cell size and cell surface were observed between S. aureus 209P and MRSA KT24. In addition, transmission electron microscopy observations of MRSA KT24 and S. aureus 209P cells at different growth phases (early exponential phase, late exponential phase and stationary phase) revealed that both S. aureus 209P and MRSA KT24 cells at early exponential phase possessed a rough outer surface and that cell-wall thickness increased gradually on growth. However, the cell wall of MRSA KT24 was clearly thicker at each phase than that of $S$. aureus 209P (Fig. 2b). Cell-wall thickness in MRSA KT24 was approximately 1.9-, 1.4- and 1.2-fold that of $S$. aureus $209 \mathrm{P}$ at early exponential phase, late exponential phase and stationary phase, respectively (Fig. 2c). Moreover, no difference in cell-wall thickness was observed in $S$. aureus ATCC 12600, ATCC 29213 and RN4220 compared with S. 
(a)

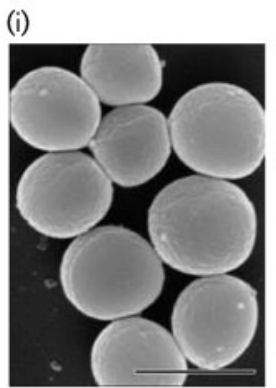

(b)

(i)

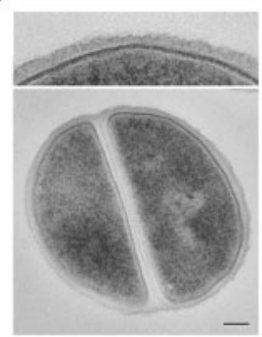

EE

(ii)

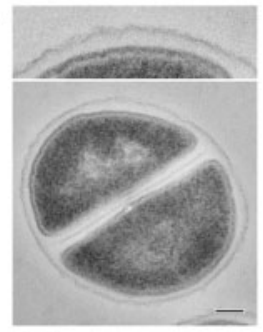

(c)

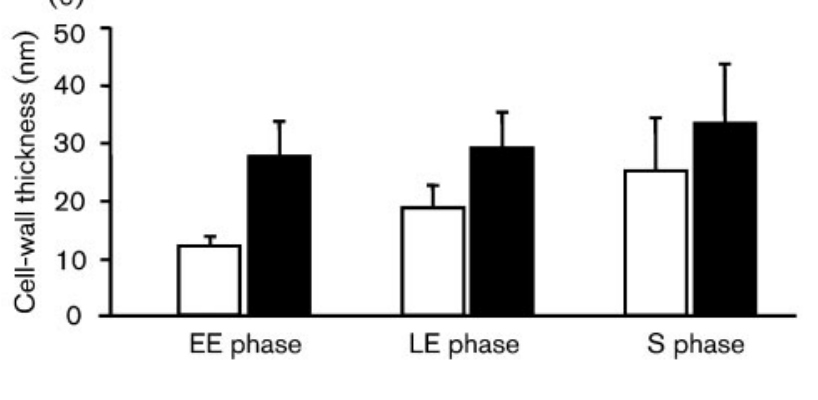

(ii)

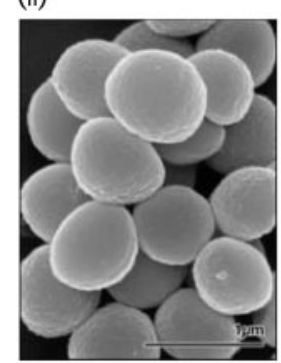

LE

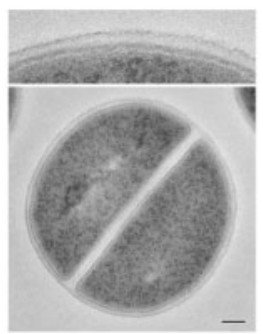

LE

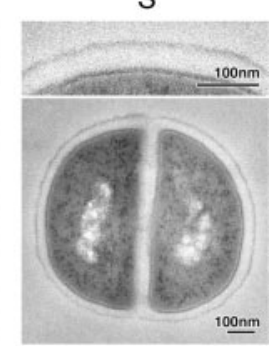

S

$\mathrm{S}$

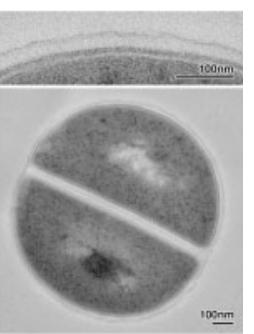

S

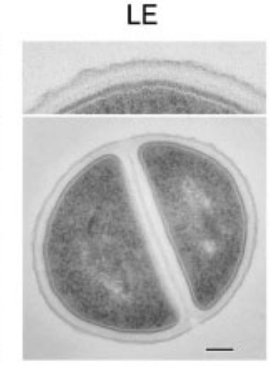

$=$

Fig. 2. Morphological analysis of MRSA KT24. Scanning electron microscopy (a) and transmission electron microscopy (b) of $S$. aureus 209P (i) and MRSA KT24 (ii) and measurements of cellwall thickness (c) at the early exponential (EE), late exponential (LE) and stationary (S) growth phases. (c) Open bars, S. aureus 209P; filled bars, MRSA KT24.

aureus 209P. The cell wall of MRSA KMP9, a multidrugresistant strain that overexpresses the NorA efflux pump, was not thickened (data not shown).

Next, we examined the morphological changes in MRSA KT24 cells exposed to sub-MIC concentrations of acriflavine. Fig. 3 shows transmission electron micrographs of representative cells cultured in SCDB with acriflavine for $4 \mathrm{~h}$. The thickened cell wall in MRSA KT24 was significantly thicker following exposure to $16 \mu \mathrm{g}$ acriflavine $\mathrm{ml}^{-1}(0.125$ MIC) (Fig. 3b). From the results of ethidium bromide accumulation and electron microscopic observation, the mechanism of acriflavine resistance in MRSA KT24 seems to involve a permeability barrier that affects cell-wall thickness.

The first paper describing the mode of cell-wall thickening in S. aureus was published by Nishino (1975), who reported that treatment with erythromycin resulted in both cell-wall thickening and a slight surface swelling in $S$. aureus. Recent studies of MRSA have shown that vancomycin is affinity trapped inside the peptidoglycan layers by false targets, serving as a physical barrier against the penetration of vancomycin molecules and resulting in vancomycin resistance (Sasatsu et al., 1989; Cui et al., 2000, 2003; Sieradzki \& Tomasz, 2003). In addition, it was shown that a thickened cell wall is responsible for vancomycin resistance in S. aureus (Cui et al., 2003). We found that MRSA KT24 had a thickened cell wall, but was susceptible to vancomycin, although we examined this using population analysis profiles (Hiramatsu et al., 1997). Therefore, cell-wall thickening in MRSA KT24 may be due to a mechanism different from that of vancomycin resistance. Further studies of the correlation between cell-wall thickness and acriflavine resistance are necessary to clarify the physiological role of cell-wall thickness in S. aureus.

(a)

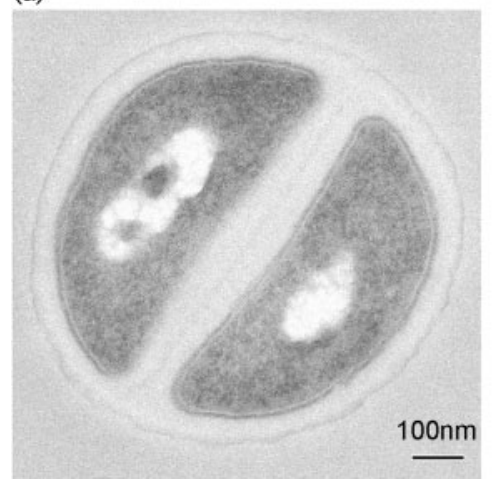

(b)

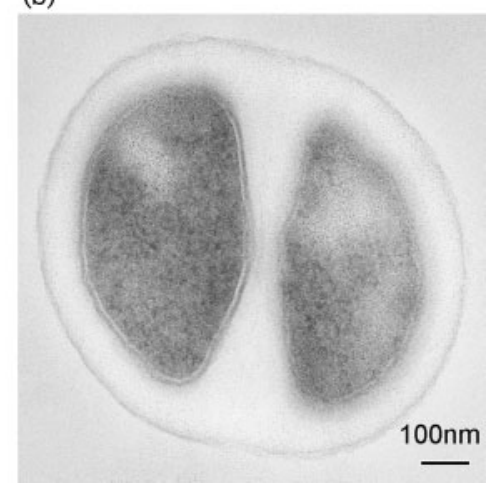

Fig. 3. Thin-section micrographs of $S$. aureus KT24 cells with and without exposure to acriflavine. (a) Normal KT24 cells; (b) KT24 cells after exposure to $16 \mu \mathrm{g}$ acriflavine $\mathrm{ml}^{-1}$ (0.125 MIC) for $4 \mathrm{~h}$. 


\section{REFERENCES}

Aeschlimann, J. R., Dresser, L. D., Kaatz, G. W. \& Rybak, M. J. (1999). Effects of NorA inhibitors on in vitro antibacterial activities and postantibiotic effects of levofloxacin, ciprofloxacin, and norfloxacin in genetically related strains of Staphylococcus aureus. Antimicrob Agents Chemother 43, 335-340.

Alam, M. M., Ishino, M. \& Kobayashi, N. (2003a). Analysis of genomic diversity and evolution of the low-level antiseptic resistance gene $s m r$ in Staphylococcus aureus. Microb Drug Resist 9, S1-S7.

Alam, M. M., Kobayashi, N., Uehara, N. \& Watanabe, N. (2003b). Analysis on distribution of high-level antiseptic resistance genes qacA and $q a c B$ in human clinical isolates of Staphylococcus aureus. Microb Drug Resist 9, 109-121.

Beenken, K. E., Dunman, P. M., McAleese, F., Macapagal, D., Murphy, E., Projan, S. J., Blevins, J. S. \& Smeltzer, M. S. (2004). Global gene expression in Staphylococcus aureus biofilm. J Bacteriol 186, 4665-4684.

Bjorland, J., Steinum, T., Sunde, M., Waage, S. \& Heir, E. (2003). Novel plasmid-borne gene qacJ mediates resistance to quaternary ammonium compounds in equine Staphylococcus aureus, Staphylococcus simulans and Staphylococcus intermedius. Antimicrob Agents Chemother 47, 3046-3052.

CLSI (2003). Methods for Dilution Antimicrobial Susceptibility Tests for Bacteria that Grow Aerobically, 6th edn. Approved Standard M7-A6. Wayne, PA: Clinical and Laboratory Standards Institute.

Cui, L., Murakami, H., Kuwahara-Arai, K., Hanaki, H. \& Hiramatsu, K. (2000). Contribution of a thickened cell wall and its glutamine nonamidated component to the vancomycin resistance expressed by Staphylococcus aureus Mu50. Antimicrob Agents Chemother 44, 22762285.

Cui, L., Xiaoxue, M., Sato, K., Okuma, K., Tenover, F. C., Mamizaka, E. M., Gemmell, C. G., Kim, M.-N., Poly, M.-C. \& other authors (2003). Cell wall thickening is a common feature of vancomycin resistance in Staphylococcus aureus. J Clin Microbiol 41, 5-14.

Gibbons, S., Oluwatuyi, M. \& Kaatz, G. W. (2003). A novel inhibitor of multidrug efflux pumps in Staphylococcus aureus. J Antimicrob Chemother 51, 13-17.

Gilbert, P. \& McBain, J. A. (2003). Potential impact of increased use of biocides in consumer products on prevalence of antibiotic resistance. Clin Microbiol Rev 16, 189-208.

Hiramatsu, K., Hanaki, H., Ino, T., Yabuta, K., Oguri, T. \& Tenover, F. C. (1997). Methicillin-resistant Staphylococcus aureus clinical strain with reduced vancomycin susceptibility. J Antimicrob Chemother 40, 135-136.

Huang, J., O'Toole, P. W., Shen, W., Amrine-Madsen, H., Jiang, X., Lobo, N., Palmer, L. M., Voelker, L., Fan, F., Gwynn, M. N. \& McDevitt, D. (2004). Novel chromosomally encoded multidrug efflux transporter MedA in Staphylococcus aureus. Antimicrob Agents Chemother 48, 909-917.

Hudson, M. C. \& Curtiss, R., III (1990). Regulation of expression of Streptococcus mutans genes important to virulence. Infect Immun 58, 464-470.

Kaatz, G. W., Seo, S. M., O'Brien, L., Wahiduzzaman, M. \& Foster, T. J. (2000). Evidence for the existence of multidrug efflux transporter distinct from NorA in Staphylococcus aureus. Antimicrob Agents Chemother 44, 1404-1406.

Mayer, S., Boos, M., Beyer, A., Fluit, A. C. \& Schmitz, F. J. (2001). Distribution of antiseptic resistance genes qacA, qacB and qacC in 497 methicillin-resistant and -susceptible European isolates of Staphylococcus aureus. J Antimicrob Chemother 47, 896-897.

McDonnell, G. \& Russell, A. D. (1999). Antiseptics and disinfectants: activity, action, and resistance. Clin Microbiol Rev 12, 147-179.

Nishino, T. (1975). An electron microscopic study of antagonism between cephalexin and erythromycin in Staphylococcus aureus. Jpn J Microbiol 19, 53-63.

Noguchi, N., Hase, M., Kitta, M., Sasatsu, M., Deguchi, K. \& Kono, M. (1999). Antiseptic susceptibility and distribution of antisepticresistance genes in methicillin-resistant Staphylococcus aureus. FEMS Microbiol Lett 172, 247-253.

Paulsen, I. T., Brown, M. H. \& Skurray, R. A. (1996). Protondependent multidrug efflux systems. Microbiol Rev 60, 575-608.

Piddock, L. J. (2006). Clinically relevant chromosomally encoded multidrug resistance efflux pumps in bacteria. Clin Microbiol Rev 19, 382-402.

Poole, K. (2002). Mechanisms of bacterial biocide and antiseptic resistance. J Appl Microbiol 92, 55S-64S.

Reifsteck, F., Wee, S. \& Wilkinson, B. J. (1987). Hydrophobicityhydrophilicity of staphylococci. J Med Microbiol 24, 65-73.

Rosenberg, M. \& Rosenberg, E. (1981). Role of adherence in growth of Acinetobacter calcoaceticus RAG-1 on hexadecane. J Bacteriol 148, 51-57.

Rouch, D. A., Cram, D. S., DiBerardino, D., Littlejohn, T. G. \& Skurray, R. A. (1990). Efflux-mediated antiseptic gene qacA from Staphylococcus aureus: common ancestry with tetracycline- and sugartransport proteins. Mol Microbiol 4, 2051-2062.

Russell, A. D. (2003). Biocide use and antibiotic resistance: the relevance of laboratory findings to clinical and environmental situations. Lancet Infect Dis 3, 794-803.

Sasatsu, M., Shima, K., Shibata, Y. \& Kono, M. (1989). Nucleotide sequence of a gene that encodes resistance to ethidium bromide from a transferable plasmid in Staphylococcus aureus. Nucleic Acids Res 17, 10103.

Sieradzki, K. \& Tomasz, A. (2003). Alterations of cell wall structure and metabolism accompany reduced susceptibility to vancomycin in an isogenic series of clinical isolates of Staphylococcus aureus. J Bacteriol 185, 7103-7110.

Suller, M. T. \& Russell, A. D. (1999). Antibiotic and biocide resistance in methicillin-resistant Staphylococcus aureus and vancomycinresistant enterococcus. J Hosp Infect 43, 281-291.

Wadström, T. (1990). Hydrophobic characteristics of staphylococci: role of surface structures and role in adhesion and host colonization. In Microbial Cell Surface Hydrophobicity, pp. 315-333. Edited by R. J. Doyle \& M. Rosenberg. Washington, DC: American Society for Microbiology.

Yamada, S. \& Matsumoto, A. (1990). Surface properties of Staphylococcus aureus affecting chemiluminescence response of human phagocytes. Microbiol Immunol 34, 809-817. 